\title{
Frequency dependence of microflows upon acoustic interactions with fluids
}

Cite as: Phys. Fluids 29, 122008 (2017); https://doi.org/10.1063/1.4999308

Submitted: 07 August 2017 . Accepted: 02 December 2017 . Published Online: 26 December 2017

Benjamin Tiller, (D) Julien Reboud, (D) Manlio Tassieri, (D) Rab Wilson, and (D) Jonathan M. Cooper

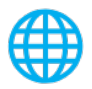

\section{ARTICLES YOU MAY BE INTERESTED IN}

Confinement of surface waves at the air-water interface to control aerosol size and dispersity

Physics of Fluids 29, 112105 (2017); https://doi.org/10.1063/1.4993793

Particle separation by phase modulated surface acoustic waves

Biomicrofluidics 11, 054115 (2017); https://doi.org/10.1063/1.5001998

Surface acoustic wave electric field effect on acoustic streaming: Numerical analysis

Journal of Applied Physics 123, 014902 (2018); https://doi.org/10.1063/1.5005849

\section{AIP Advances Fluids and Plasmas Collection}




\title{
Frequency dependence of microflows upon acoustic interactions with fluids
}

\author{
Benjamin Tiller, ${ }^{\text {a) }}$ Julien Reboud, Manlio Tassieri, Rab Wilson, and Jonathan M. Cooper \\ Division of Biomedical Engineering, School of Engineering, University of Glasgow, Rankine Building, \\ Oakfield Avenue, G12 8LT Glasgow, United Kingdom
}

(Received 7 August 2017; accepted 2 December 2017; published online 26 December 2017)

\begin{abstract}
Rayleigh surface acoustic waves (SAWs), generated on piezoelectric substrates, can interact with liquids to generate fast streaming flows. Although studied extensively, mainly phenomenologically, the effect of the SAW frequency on streaming in fluids in constrained volumes is not fully understood, resulting in sub-optimal correlations between models and experimental observations. Using microfluidic structures to reproducibly define the fluid volume, we use recent advances modeling the body force generated by SAWs to develop a deeper understanding of the effect of acoustic frequency on the magnitude of streaming flows. We implement this as a new predictive tool using a finite element model of fluid motion to establish optimized conditions for streaming. The model is corroborated experimentally over a range of different acoustic excitation frequencies enabling us to validate a design tool, linking microfluidic channel dimensions with frequencies and streaming efficiencies. We show that in typical microfluidic chambers, the length and height of the chamber are critical in determining the optimum frequency, with smaller geometries requiring higher frequencies. Published by AIP Publishing. https://doi.org/10.1063/1.4999308
\end{abstract}

\section{INTRODUCTION}

Surface acoustic waves (SAWs) can be used to actuate liquids and produce ultrasonic radiation forces, so enabling a wide variety of phenomena, including the sorting of particles or cells based upon their physical properties, ${ }^{1}$ the nebulisation of droplets as a potential drug delivery platform, ${ }^{2}$ the use of acoustic streaming to drive efficient fluid mixing in droplets, ${ }^{3}$ and the actuation of fluid flows through microfluidic networks as an efficient alternative to "bulky" hydrodynamic pumps. ${ }^{4,5}$ However, despite the large number of such studies of SAWs within microfluidic devices, the dependency of the induced fluid streaming on the applied acoustic frequency and the geometry of the microsystems used is still not fully understood.

In many areas of application, ${ }^{1-5}$ there is a requirement to create strong streaming with high flow velocities which in itself can lead to practical limitations in both maintaining the integrity of devices ${ }^{6}$ and in the amount of power required to drive the acoustic transducers (before undesired local heating effects appear). One motivation therefore for optimising streaming is to generate higher velocity flows for a given power, ${ }^{7-10}$ by quantitatively exploring the dependence of fluid flow on SAW frequency.

The concept of optimising the steady fluid velocity created from oscillating acoustic waves through acoustic streaming was first shown in a theoretical scaling analysis ${ }^{11}$ while studying the propagation of a plane longitudinal wave through a cylindrical pipe. The authors demonstrated that the average

\footnotetext{
a) Present address: Department of Electronic and Electrical Engineering, University of Strathclyde, Royal College Building, 204 George St., Glasgow G1 1XW, United Kingdom.
}

bulk streaming velocity scales exponentially with the frequency when the radius of the pipe was smaller than the acoustic wavelength. Although similar scaling arguments should exist for streaming generated by SAWs, the translation of this analysis into practical applications has been limited. Specifically, when a SAW refracts into a fluid, the acoustic field generated is asymmetric, which introduces more complexity than when the field is generated by a plane bulk acoustic wave, rendering accurate predictions more difficult.

Studies on SAW-induced streaming in sessile droplets have provided methods for modelling the distribution of the acoustic field within the fluid, by considering the attenuation length of the SAW beam as a scaling parameter. ${ }^{12,13}$ This approach however ignores the effect of the viscous attenuation due to the fluid, preventing close agreement with the experimental evidence. A more accurate description of the scaling laws governing the SAW-induced streaming in microfluidic channels ${ }^{10,14}$ gives an expression for the acoustic pressure field based on both the viscous damping of the fluid itself and the damping of the SAW by the fluid.

In the context of typical microfluidic structures, these expressions translate to the physical constraints of the geometry on the body of the liquid, such as the height of microchannels. When a channel is smaller than the attenuation length of the SAW, the magnitude of streaming flows is significantly decreased. Recent advances have taken the so-called "anechoic corner" into account and shown good agreement between simulation and experiment, ${ }^{15,16}$ but only over a limited range of frequencies, preventing the generation of significant insight into the interplay between acoustic frequency and streaming efficiency.

The effect of frequency on SAW streaming in fluids has also been investigated without explicit derivation of the 
acoustic field and showed good agreement with a streaming model within large volumes. ${ }^{17}$ However, the conclusions do not extend to microfluidic systems, where the length scales are often many times smaller than the attenuation length of the acoustic beam. Moreover, the effect of frequency on SAWinduced flows used for mixing different fluids in droplets has led to the scaling argument that higher frequencies are able to drive faster mixing in small droplets. ${ }^{18}$

In this work, we expand upon the approaches for modelling the acoustic field in microfluidics channels ${ }^{10,14,15}$ and build a simple expression of the body force, taking into account the anechoic corner as well as the damping coefficients of both the SAW and the viscous damping of the fluid. We demonstrate, for the first time, how these expressions can be used to fully analyse the scaling of SAW-induced acoustic streaming with the acoustic frequency.

We adopt this expression to predict SAW streaming in a fluid using a finite element model and we quantitatively validate it by comparing the simulation results with microparticle imaging velocimetry ( $\mu \mathrm{PIV})$ measurements of streaming in a chamber across a range of frequencies. This allows us to provide a predictive tool by which to design acoustofluidic devices, by determining the optimum frequency for driving streaming in different microfluidic volumes. Contrary to the iterative approaches currently used during the design of many SAW-based microfluidic devices, we demonstrate that the frequency should be tailored to the length scale of the microfluidic device and that frequencies $>500 \mathrm{MHz}$, i.e., outside of the usual used range (1-10 MHz), will drive the fastest streaming for microfluidic length scales ranging from 10 to $100 \mu \mathrm{m}$.

\section{THEORY}

Full numerical simulations to model acoustic fields in both the fluid and solid domains together with the resulting acoustic streaming have previously been reported. ${ }^{18,19}$ These provide physically accurate models, but at the expense of high complexity and high computational demands, preventing more useful parametric studies to explore this design space. Consequently, partial methods have been developed, where an analytical approximation for the acoustic field in the fluid due to the SAW can be derived to calculate the body force using Nyborg's streaming equations. In a further iteration, the body force has been used to calculate streaming numerically. ${ }^{13,14}$

We now provide a new explicit expression of the body force generated by the SAW-induced acoustic field, enabling a generic predictive framework to optimise the frequency dependent magnitude of streaming flows. We derive an expression for this body force, $\mathbf{F}_{\mathbf{b}}$, using a method of successive approximations to solve the Navier Stokes equation, ${ }^{20}$ which we subsequently use to calculate the resulting steady state streaming in a fluid,

$$
\mathbf{F}_{\mathbf{b}}=\rho_{f}\langle\mathbf{u} \cdot \nabla \mathbf{u}+\mathbf{u} \nabla \cdot \mathbf{u}\rangle,
$$

where $\mathbf{u}$ is the velocity field in the fluid due to the acoustic oscillatory motion and $\rho_{f}$ is the fluid density. It is worth noting that this theoretical framework makes assumptions that limit its application to slow fluid streaming, i.e., $\operatorname{Re}<1$ and $|\mathbf{u}|>|\xi|$, where the Reynolds number is defined as $R e=\rho|\xi| l / \mu$, with $\xi$ as the static streaming velocity and $l$ as the microfluidics' characteristic length scale. ${ }^{21}$

\section{ACOUSTIC FIELDS IN FLUIDS DUE TO RAYLEIGH ACOUSTIC WAVES}

When a SAW such as a Rayleigh wave is generated on a solid substrate, it propagates along the surface as an elliptically polarized wave, with the displacement amplitude decaying into the substrate. Upon reaching a fluid such as a droplet or a fluidic stream placed on its path, the SAW amplitude is exponentially damped, propagating as a "leaky surface wave" at the fluid/substrate interface due to the generation of a compression wave in the fluid. This compression wave itself propagates at the Rayleigh angle, ${ }^{22} \theta_{R}=\sin ^{-1}\left(v_{f} / v_{S A W}\right)$, where $v_{f}$ is the propagation velocity of the compression wave in the fluid and $v_{S A W}$ is the propagation velocity of the Rayleigh wave on the substrate.

In order to derive an expression of the SAW-induced body force within the fluid, we consider the acoustic energy loss along a specific two-dimensional path to reach a point $P$ in the fluid (as a cross section of the volume). The path is drawn as a sum of a distance $S$ in the direction of SAW propagation at the solid/liquid interface and a distance D longitudinal to the wave propagation at the Rayleigh angle, ${ }^{10}$ as shown schematically in Fig. 1(a). The total attenuation through that path is $e^{-D \alpha_{f}} e^{-s \alpha_{S A W}}$, where $\alpha_{f}=\left(b \omega^{2}\right) /\left(2 \rho_{f} v_{f}^{3}\right)$ is the attenuation of a plane wave in a viscous fluid, $\alpha_{S A W}=\rho_{f} v_{f} /\left(\rho_{s} v_{S A W} \lambda_{S A W}\right)$ is the attenuation of a fluid-damped Rayleigh SAW, ${ }^{17} \rho_{f}$ and $\rho_{s}$ are the fluid and substrate densities, respectively, $\lambda_{S A W}$ is the SAW wavelength, $\omega$ is the angular frequency, and $b=\left(\frac{4}{3} \mu+\mu^{\prime}\right)$, where $\mu^{\prime}$ is the bulk viscosity. Accordingly, the velocity field in 2 dimensions is written as

$$
\begin{aligned}
& u_{y}=\omega A \tan \left(\theta_{R}\right) e^{i\left(\omega t-k_{y} y-k_{z} z\right)} e^{\left(-\left|y-z \tan \left(\theta_{R}\right)\right| \alpha_{S A W}\right)-z \cos ^{-1}\left(\theta_{R}\right) \alpha_{f}} \\
& u_{z}=\omega A e^{i\left(\omega t-k_{y} y-k_{z} z\right)} e^{\left(-\left|y-z \tan \left(\theta_{R}\right)\right| \alpha_{S A W}\right)-z \cos ^{-1}\left(\theta_{R}\right) \alpha_{f}}
\end{aligned}
$$

where $k_{y}=k \sin \left(\theta_{R}\right)$ and $k_{z}=k \cos \left(\theta_{R}\right)$ are wavenumbers in $\mathrm{y}$ and $\mathrm{z}$ with $k=(2 \pi f) / v_{f}$.

Here we take into consideration the whole volume of the fluid, including microstructures [such as an anechoic corner, shown in Fig. 1(a)], enabling us to build an accurate description of an acoustically pumped microfluidic system. ${ }^{15} \mathrm{We}$ do not consider the reflections off the walls of the microchannels (which are most often made of elastomeric materials with a low acoustic impedance, and which do not generate significant reflections). It is important to note that, where other materials are used in the construction of the microfluidic network (such as glass or silicon, for example), the contribution of such reflections will be significant and will need to be considered systematically.

The anechoic corner produced by the angled path of the sound beam can be further defined by specifying that $u_{y}$ and $u_{z}=0$ when $z>y /\left(\tan \left(\theta_{R}\right)\right)$. The body force on the fluid due to the sound field can then be calculated by replacing the full 

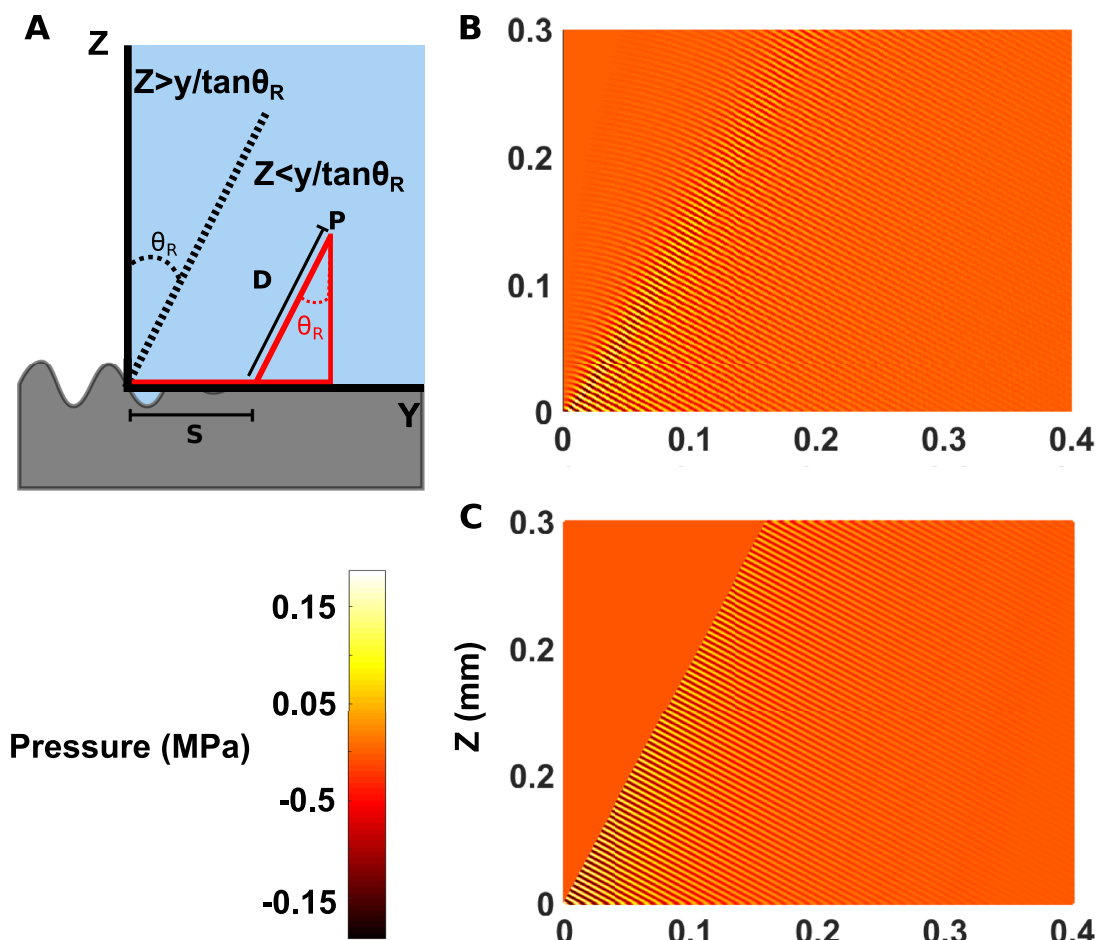

\section{0}

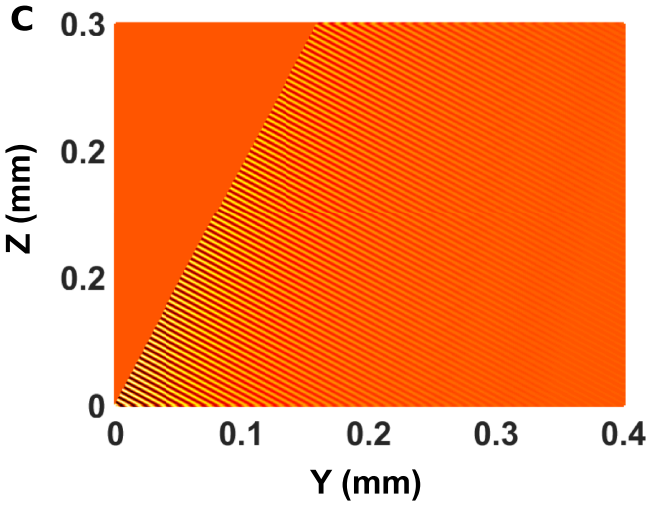

FIG. 1. (a) Schematic representation of a chosen path taken by the acoustic wave for derivation of the body force. A Rayleigh SAW travels along the surface of a substrate (from the left to right) until it reaches a fluid volume (blue shaded area). The SAW then propagates along the fluid-substrate interface and radiates part of its energy into the fluid. This energy propagates through the fluid as a longitudinal compression wave, at a specific Rayleigh angle to the SAW direction. (b) Finite element simulation of the pressure field in the fluid due to a $342 \mathrm{MHz}$ SAW. (c) shows the pressure field predicted by the analytical expression for the velocity field in Eq. (2). expressions of $u_{\mathrm{y}}$ and $u_{z}$ into the body force equation

$$
\begin{aligned}
& F_{y}=\rho_{f}\left(\tan \left(\theta_{R}\right) k_{y i}+k_{z i}\right) A^{2} \omega^{2} \tan \left(\theta_{R}\right) e^{2\left(k_{y i}+k_{z i}\right)}, \\
& F_{z}=\rho_{f}\left(\tan \left(\theta_{R}\right) k_{y i}+k_{z i}\right) A^{2} \omega^{2} e^{2\left(k_{y i}+k_{z i}\right)},
\end{aligned}
$$

where $k_{y i}=-\alpha_{S A W}$ and $k_{z i}=-\cos ^{-1}\left(\theta_{R}\right) \alpha_{f}+\tan \left(\theta_{R}\right) \alpha_{S A W}$.

This analytical expression for the acoustic field was explored using a COMSOL simulation of the pressure field in a $2 \mathrm{D}$ rectangular fluid domain due a leaky SAW propagating vibrations along the bottom boundary. A comparison between the finite element simulation and the analytical expression for a $342 \mathrm{MHz}$ SAW vibration is shown in Figs. 1(b) and 1(c) (results for $43 \mathrm{MHz}$ and $87 \mathrm{MHz}$ are also shown in Fig. S2 of the supplementary material).

\section{EXPERIMENTAL SECTION}

Simulations in Comsol Multiphysics 3.5a (acoustic module) were compared with experimental measurements of SAW streaming in microfluidic chambers, measured with $\mu$ PIV. The microchamber [Fig. S1(a) of the supplementary material] was made using the elastomeric polymer poly(dimethyl)siloxane (PDMS), fabricated using standard soft lithographic methods with dimensions $0.4 \times 0.4$ $\times 0.15 \mathrm{~mm}$ (XYZ). An associated microfluidic channel network was moulded on the surface of the substrate in the path of the SAW. The SAW was generated on a piezoelectric Y-cut lithium niobate substrate by applying an alternating electric field to an inter-digitated transducer (IDT). The width of each finger of the IDTs and the distance between each finger were equal. Each IDT consisted of 40 pairs of fingers, and aperture and finger widths were, respectively, $2 \mathrm{~mm}$ and $2.5 \mu \mathrm{m}$ for $342 \mathrm{MHz}, 6 \mathrm{~mm}$ and $10 \mu \mathrm{m}$ for $87 \mathrm{MHz}$, and $6 \mathrm{~mm}$ and $20 \mu \mathrm{m}$ for $43 \mathrm{MHz}$, respectively. A quarter wavelength reflector was also included behind the IDTs to partially reflect back some of the lost wave energy. ${ }^{23}$ The IDTs were fabricated using standard photolithography, metal evaporation, and lift-off to produce microelectrodes with $10 \mathrm{~nm}$ of $\mathrm{Ti}$ (as an adhesion layer) capped with $100 \mathrm{~nm}$ of $\mathrm{Au}$, as previously described. ${ }^{24}$

To minimize the damping from the elastomeric polymer and also to control the position of the SAW beam in the chamber, a further "beam tunnel" was included into the channel network design [see Figs. S1(a) and S1(b) of the supplementary material] with a thin $(100 \mu \mathrm{m})$ PDMS wall, minimizing the energy losses. By designing the width and position of the tunnel relative to the fluid filled channels, the SAW beam could be spatially positioned within the chamber, allowing the control of the direction and shape of the induced fluid streaming. This was used to create a stable anticlockwise vortex in the chamber, measured with $\mu \mathrm{PIV}$, using $1 \mu \mathrm{m}$ diameter fluorescent polystyrene beads as tracers within the fluid flow (in this case, water). A $10 \times$ objective lens focused a laser pulse onto a plane perpendicular to the $\mathrm{z}$ direction in the chamber (see the supplementary material for details).

Scanning laser Doppler vibrometry (UHF-120 Ultra High Frequency Vibrometer, Polytec $\mathrm{GmbH}$ ) was used to measure the amplitude of the vertical displacement in the SAW beam and avoid any discrepancies that might arise when using the exciting rf power. In particular, we used these measurements to relate the observed liquid streaming to the amplitude of the SAW beam in the chamber (the beam amplitude was found to approximate a Gaussian or double Gaussian distribution in $x$, depending on frequency, Fig. 2). This information was incorporated into the simulation by expressing the amplitude in the body force as a function of $\mathrm{x}$. 
A $342 \mathrm{MHz}$
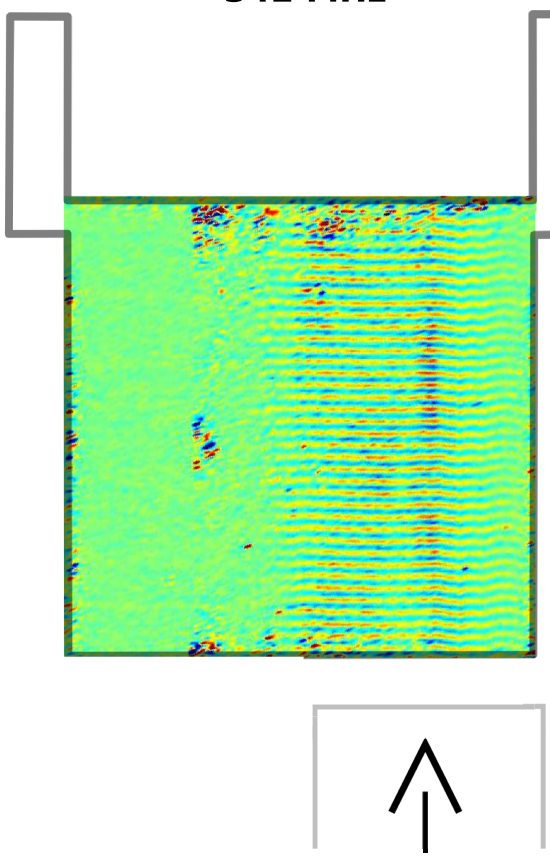

SAW Beam
B

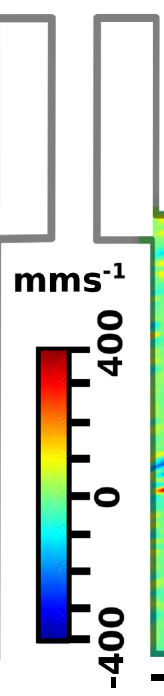

\section{$87 \mathrm{MHz}$}

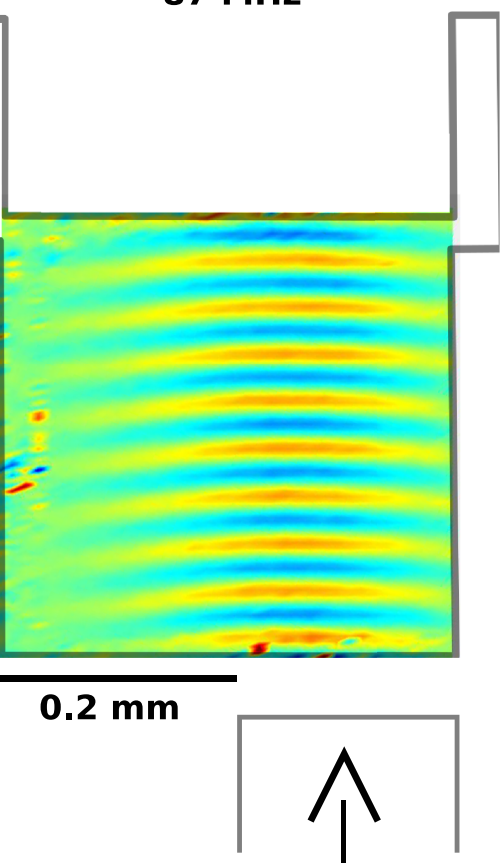

D

SAW Beam
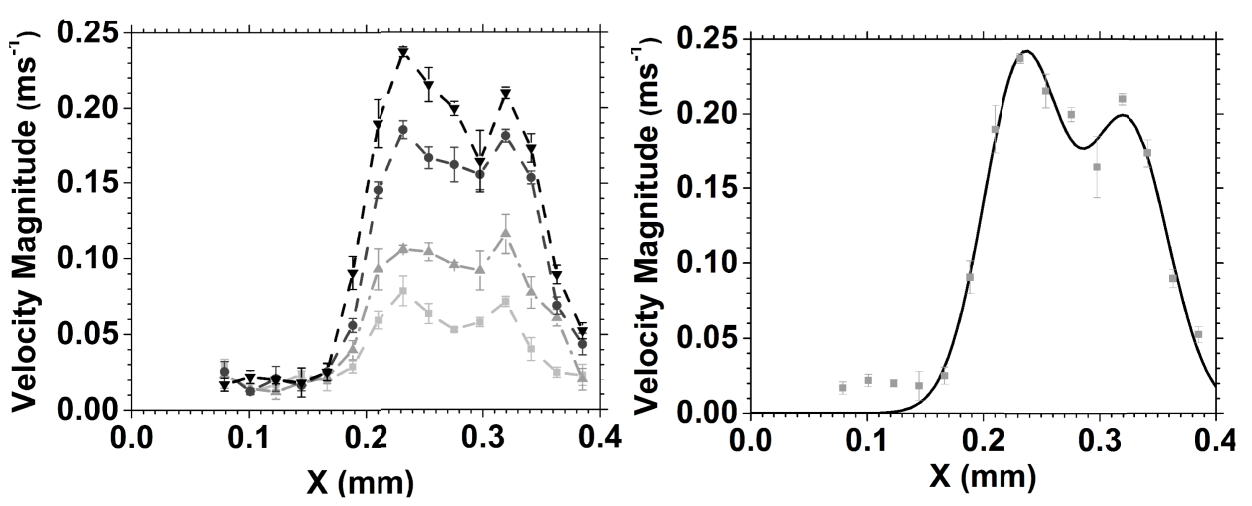

FIG. 2. Vibrometer scans of the surface waves in the square micro-chamber for $342 \mathrm{MHz}$ (a) and $87 \mathrm{MHz}$ (b). Most of the SAW energy is confined to half of the chamber (along the $\mathrm{x}$-axis) due to the guiding effect of the beam tunnel. This can also be seen to act like a diffraction slit for the lower frequency $87 \mathrm{MHz}$ SAW, exhibiting some wave spreading, which was not seen at $342 \mathrm{MHz}$. The colour scale shows the velocity amplitude of the SAW beam. (c) shows a cross section of a $342 \mathrm{MHz}$ SAW beam along the $\mathrm{x}$ axis after the SAW has entered the chamber for different electrical input powers (from light to dark, $0.03,0.10$, $0.32,1.00 \mathrm{~W}$ ). (d) A double Gaussian peak fit for the cross section at $1 \mathrm{~W}$ $\left(\mathrm{R}^{2}=0.978\right)$. This fit was used to describe the amplitude profile of the SAW beam in the simulation.

\section{RESULTS AND DISCUSSION}

We validated the accuracy of the new model by comparing simulations with $\mu \mathrm{PIV}$ measurements at different heights in the microchannel. At $\mathrm{h}=25 \mu \mathrm{m}$ (Fig. 3), the body force due to the SAW pushes the fluid up through the chamber. At $\mathrm{h}=50 \mu \mathrm{m}$ and $\mathrm{h}=75 \mu \mathrm{m}$, there is an anti-clockwise flow in the chamber due to the unbalanced body force, which only exists in the right-hand side of the chamber (see Figs. S4 and S5 of the supplementary material).

Higher frequency SAW beams generated faster streaming, with the average streaming magnitude over the whole chamber for the $342 \mathrm{MHz}$ beam being more than an order of magnitude greater than for $43 \mathrm{MHz}$. The experimental measurements are in close quantitative agreement with the simulation results, as shown in Fig. 4.

The good agreement with experimental data allowed us to explore the model as a design tool, linking microfluidic channel dimensions with frequencies and streaming efficiencies. Simulations (Fig. 5) were used to explore the geometrical parameter space and allowed us to choose the most suitable conditions for a specific application, depending on SAW streaming. Results show a strong dependency of the streaming magnitude as a function of the SAW frequency; with frequencies between 270 and $374 \mathrm{MHz}$ driving the fastest streaming in the case of a microchannel having dimensions of $0.4 \times 0.3 \times 0.3 \mathrm{~mm}(\mathrm{XYZ})$. Hence, this new implementation provides the opportunity of predicting an "optimum" frequency to drive SAW streaming in a given microfluidic chamber.

When $l>1 / \alpha_{S A W}$, the SAW attenuates completely before reaching the end of the chamber. In this case, the optimum frequency, $F_{\max }$, was determined by considering three factors: the dimensions of the chamber (relating to the compressional wave); the power radiated into the fluid; as well as the viscous and inertial losses. At higher frequencies, the beam attenuates faster into the fluid so that more energy is transferred to it, whereas, at lower frequencies a proportion of the energy available for streaming is lost mainly due to refraction through the roof and sidewalls of the chamber. 
A

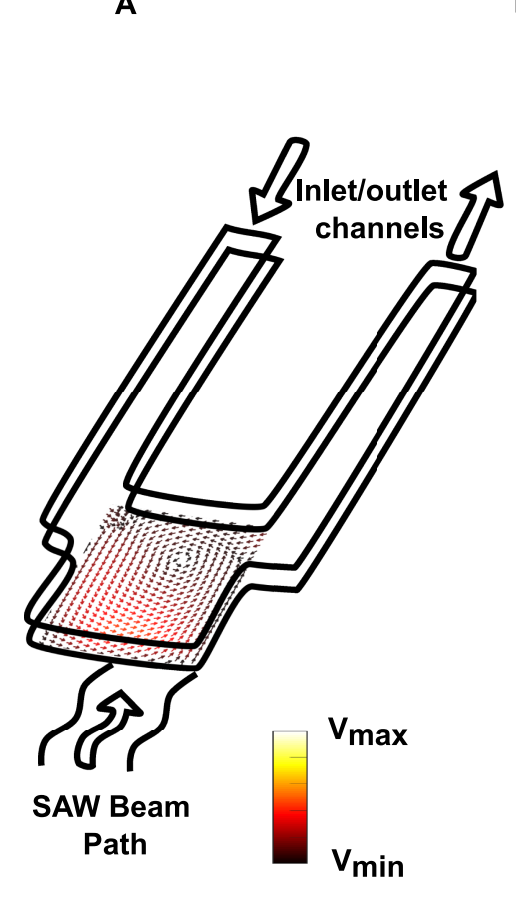

B

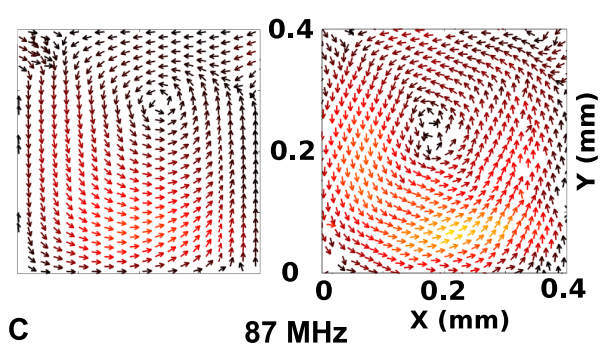

$43 \mathrm{MHz}$

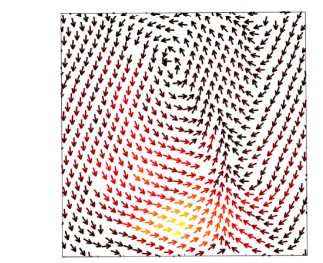

D

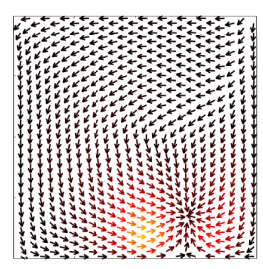

Simulation

\section{$342 \mathrm{MHz}$}

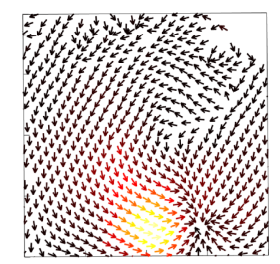

FIG. 3. Quiver plots of the in-plane fluid velocity at different frequencies for a cross-sectional height, $\mathrm{h}$, of $25 \mu \mathrm{m}$. (a) Schematic representation to aid visualisation. The quiver plots are organized in pairs, comparing simulated (left) and measured (right) results for 43, 87, and $342 \mathrm{MHz}$ [(b)-(d), respectively] with the colour scales used to make easy visual comparison. Figures S3-S5 of the supplementary material further show comparisons between the simulation and measurement over a range of different chamber heights $(50,75$, and $100 \mu \mathrm{m})$.

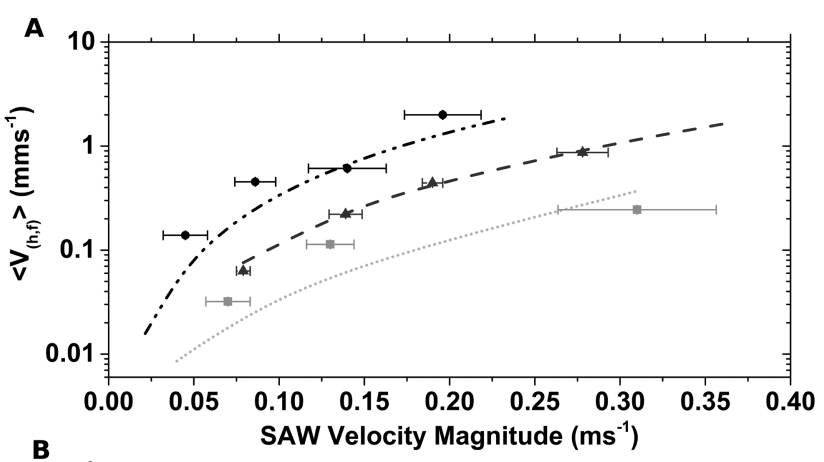

$$
\text { B }
$$

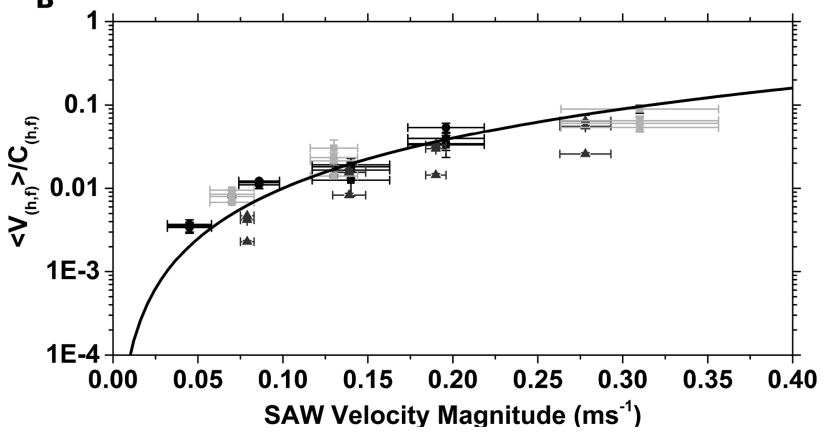

FIG. 4. (a) Simulation results of streaming velocities as a function of the magnitude of SAW velocity at a chamber height of $75 \mu \mathrm{m}$ (averaged over the whole chamber, with the standard deviation shown as an error bar). For equal amplitude particle velocities, the higher frequency SAW beams generated the fastest average streaming (dark to light: 342, 87, and $43 \mathrm{MHz}$ ). (b) Average measured streaming velocity for all chamber heights and frequencies studied, normalised to SAW amplitude velocity, $u_{z(z=0, x=0)}^{2}$. Each experimental data set was divided by a factor $\mathrm{C}(\mathrm{h}, \mathrm{f})$ determined by fitting each simulation curve to $V_{(h, f)}=C_{(h, f)} u_{z(z=0, x=0)}^{2}$. This allows an immediate comparison of the agreement of all the measurements with the theoretical prediction. The colour scale is the same as in (a).
On the other hand, the power radiated by the SAW into the fluid compressional wave is inversely proportional to frequency: ${ }^{17}$

$$
P=\int_{0}^{l} \rho w v_{f} \eta_{0}^{2} e^{-2 \alpha_{s a w} Y} d Y
$$

Where $l$ is the length of the chamber, $w$ is the width of the SAW beam and $\eta_{0}$ is the initial velocity amplitude of the SAW. The viscous and inertial effects also depend on frequency: at lower frequencies inertia is negligible and the fluid velocity scales with $f^{2}$, as described in the classical equations first derived by Nyborg; ${ }^{20}$ whereas, at higher frequencies the streaming flows are better described as a StuartLighthill type streaming jet. ${ }^{25}$ In this regime, the fluid inertia becomes significant and the fluid velocity no longer scales with $f^{2}$.

These factors enable an interpretation of the behaviour observed in Fig. 5, where, at low frequencies, the acoustic beam is longer than the path to the chamber roof $\left(h<\alpha_{f}^{-1} \cos \left(\theta_{R}\right)\right)$ and the process is governed by the height of the chamber. As frequency increases, the length of the beam decreases, providing more energy into the fluid to drive streaming. When the acoustic beam becomes much shorter than the height of the chamber, the streaming velocity decreases due to the onset of streaming jet-like behaviour.

The same concept can be applied to the other dimensions of the chamber. For example, when $l<1 / \alpha_{S A W}$, a fraction of the acoustic energy is lost to the back end of the chamber, thus decreasing the total power radiated into the fluid. This shifts the optimum frequency towards 
A

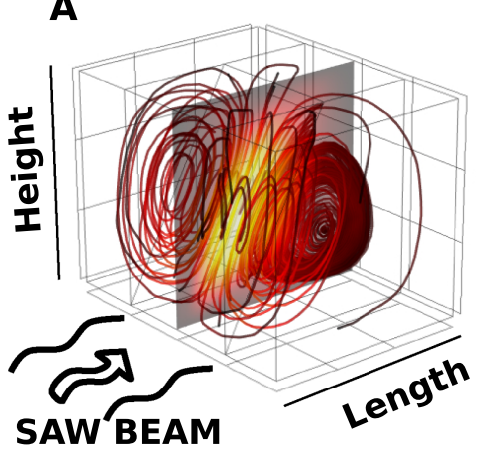

C
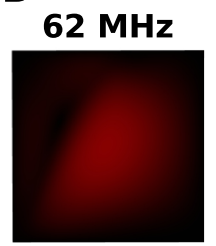

$374 \mathrm{MHz}$

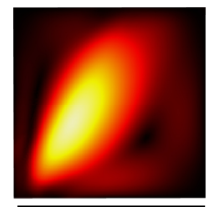

$470 \mathrm{MHz}$

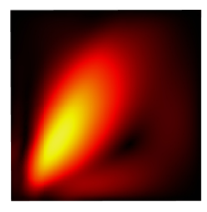

$530 \mathrm{MHz}$

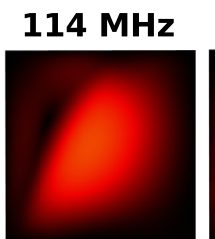

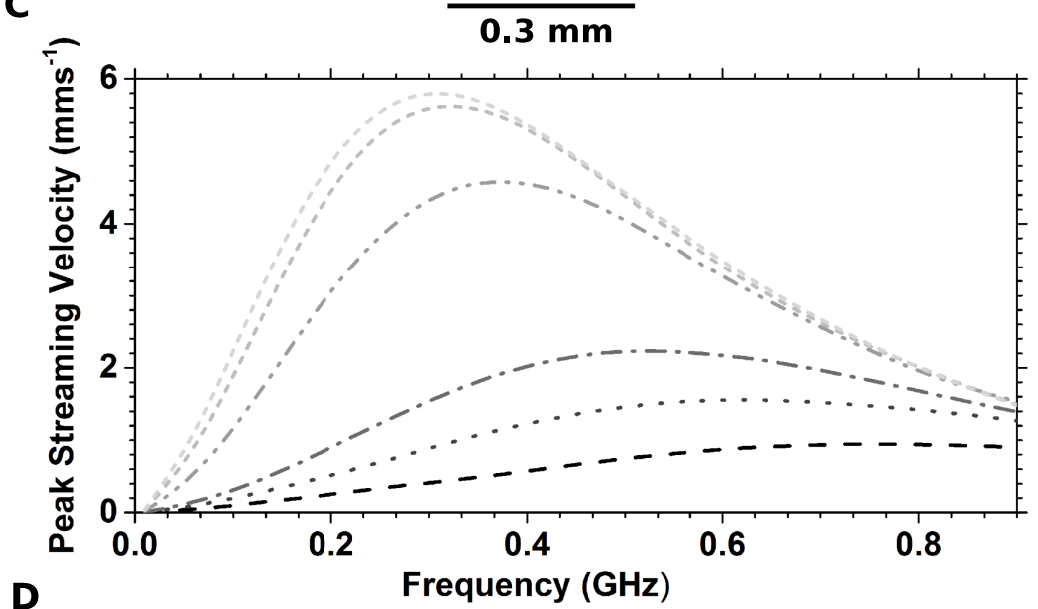

B

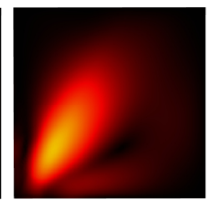

$270 \mathrm{MHz}$

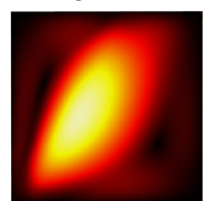

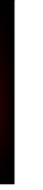

\section{.}

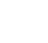

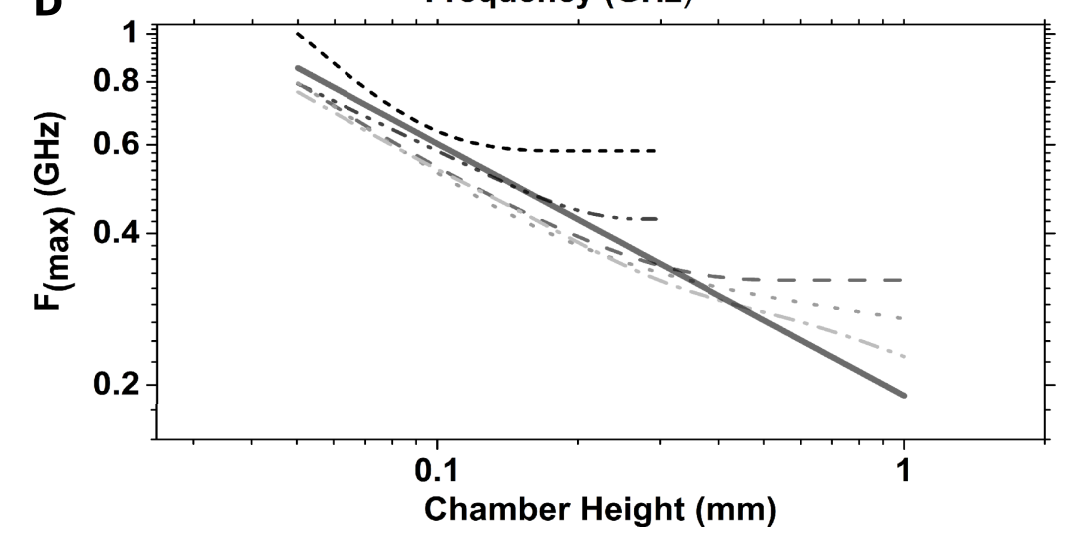

FIG. 5. (a) Streamline plot of the fluid flow in a chamber with dimensions $0.4 \times 0.3 \times 0.3 \mathrm{~mm}(\mathrm{XYZ})$, where the width of the SAW beam is incident over $0.1<\mathrm{x}<0.3 \mathrm{~mm}$. (b) Cross sections of the fluid flow for a number of different frequencies keeping the initial SAW velocity amplitude constant at $1 \mathrm{~ms}^{-1}$; the colour scale is the same for each cross section. The spatial position of the cross section in the chamber is shown in (a). (c) Peak streaming velocity against frequency for chamber volumes with dimensions $0.3 \times 0.3 \times \mathrm{h} \mathrm{mm}(\mathrm{XYZ})$ (from light to dark, $h=0.400,0.300$, $0.200,0.100,0.075,0.050 \mathrm{~mm}$ ). (d) The optimum frequencies are related to the chamber height and length. When the length of the chamber is much larger than $1 / \alpha_{S A W}$; it is the height of the chamber that determines the optimum frequency (from dark to light $l=0.05,0.10$, $0.20,0.40,1.50 \mathrm{~mm})$. The solid line is added as a visual guide for this scaling relationship $\left(h=\alpha_{f}^{-1} \cos \left(\theta_{R}\right)\right)$. As the ratio of the chamber's length to height increases, the optimum frequencies collapse on this relationship. higher values, while keeping all other parameters constant [Fig. 5(d)].

It is useful to note also that, at low frequencies, our results are in agreement with those reported in the literature, ${ }^{9,17}$ but differ at high frequencies because of differences in normalisation methods 9 and modelling approaches. ${ }^{17}$ In particular, in an unbounded regime, Dentry et al. ${ }^{17}$ predicted a constant relationship between frequency and peak streaming velocity for frequencies up to $1 \mathrm{GHz}$, which is in contrast with our simulation results that show a decrease in peak streaming velocity (Fig. 5, confirmed also for unbounded cases in Fig. S6 of the supplementary material). We attribute these differences to the onset of inertial losses in the fluid at high frequencies, which are not taken into account by Dentry et $\mathrm{al}$. when using the laminar jet model. Our understanding is supported by the work of Eisener et al., ${ }^{25}$ in which they observed that by using a "piston-type" acoustic transducer, the peak streaming velocity shows a decreasing trend at frequencies higher than $600 \mathrm{MHz}$. Moreover, in the case of SAW streaming, an additional $f^{-1}$ scaling of the power radiated in the fluid [Eq. (4)] imposes a sharper decrease of the peak streaming velocity with increasing frequency.

As an example of how this new understanding can drive the design process, Fig. 6(a) was created by interpolating between 36 simulations over a range of chamber heights and lengths spanning from 0.05 to $0.4 \mathrm{~mm}$, enabling to select the optimum frequency for driving the fastest streaming. In agreement with the previous results, the figure shows that as the dimensions are increased, the optimum frequency to generate fast streaming flows becomes lower. It also shows that the channel height has a more significant impact upon the frequency than the horizontal dimensions, thus providing a clear guide to select specific frequencies for a given geometry. Figure 6(b) also shows peak streaming velocity generated 
A

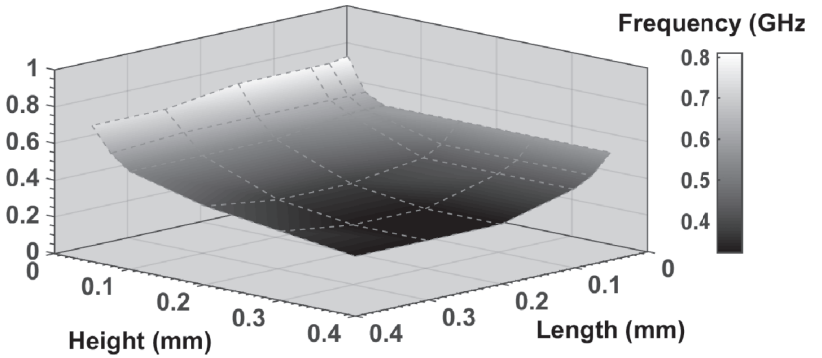

B

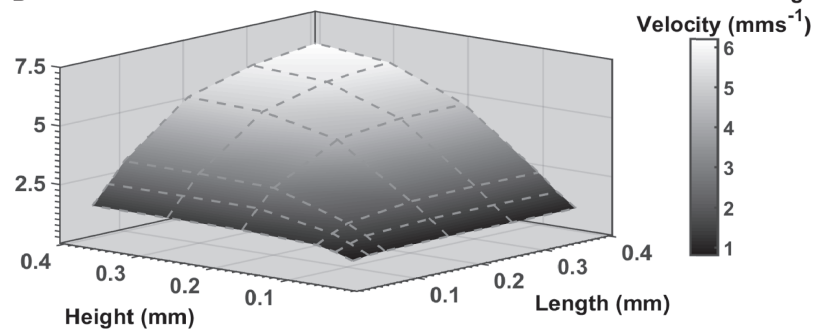

FIG. 6. (a) Optimum frequency for driving the fastest streaming as a function of the length and height of the microfluidic chamber within the range 0.05$0.4 \mathrm{~mm}$. (b) Peak streaming velocity for the optimum frequency as a function of length and height. These plots are a linear interpolation of the optimum frequency from 36 simulations. A 2D visualisation is available in Fig. S7 of the supplementary material.

by the optimum frequency for different chamber dimensions, where smaller chamber dimensions limit the maximum streaming velocity that is achievable.

\section{CONCLUSIONS}

We present an expression of the body force exerted on a fluid through its mechanical interaction with a surface acoustic wave. A simulation for the resulting acoustic streaming was constructed by considering the compression wave radiated into the fluid by the SAW and relating this to the classic streaming equations popularised by Nyborg. This was tested experimentally and used to provide a predictive framework for optimising the magnitude of frequency dependent microflows generated by acoustic streaming. We demonstrate the utility of this approach by simulating flows in different microfluidic structures to optimise the acoustic frequency for generating maximum flow rates. We further show a good quantitative agreement with experimental data, allowing us to explore the model's use as a design tool, linking microfluidic channel dimensions with frequencies and streaming efficiencies.

\section{SUPPLEMENTARY MATERIAL}

See supplementary material for Figs. S1-S7 and more details on PIV measurement and COMSOL simulations. All data associated with this manuscript is available open access at https://doi.org/10.5525/gla.researchdata.560.

\section{ACKNOWLEDGMENTS}

The authors are grateful for EPSRC support (No. EP/I017887/1-Proxomics) and to Martin Hyde and the
EPSRC equipment loan pool for the $\mu$ PIV system. J.M.C. acknowledges EPSRC Fellowship (No. EP/K027611/1) and the ERC Advanced Investigator Award (No. 340117Biophononics). J.R. acknowledges a Lord Kelvin and Adam Smith research fellowship (University of Glasgow). M.T. acknowledges support from the Royal Academy of Engineering/EPSRC Fellowship (No. 10216/101).

${ }^{1}$ J. Shi, D. Ahmed, X. Mao, S.-C. S. Lin, A. Lawit, and T. J. Huang, "Acoustic tweezers: Patterning cells and microparticles using standing surface acoustic waves (SSAW)," Lab Chip 9, 2890 (2009).

${ }^{2}$ A. Qi, J. R. Friend, L. Y. Yeo, D. A. V. Morton, M. P. McIntosh, and L. Spiccia, "Miniature inhalation therapy platform using surface acoustic wave microfluidic atomization," Lab Chip 9, 2184 (2009).

${ }^{3}$ A. Wixforth, C. Strobl, C. Gauer, A. Toegl, J. Scriba, and Z. Guttenberg, "Acoustic manipulation of small droplets," Anal. Bioanal. Chem. 379, 982 (2004).

${ }^{4}$ L. Schmid, A. Wixforth, D. A. Weitz, and T. Franke, "Novel surface acoustic wave (SAW)-driven closed PDMS flow chamber," Microfluid. Nanofluid. 12, 229 (2012).

${ }^{5}$ M. B. Dentry, J. R. Friend, and L. Y. Yeo, "Continuous flow actuation between external reservoirs in small-scale devices driven by surface acoustic waves," Lab Chip 14, 750 (2014).

${ }^{6}$ H.-F. Pang, Y. Q. Fu, L. Garcia-Gancedo, S. Porro, J. K. Luo, F. Placido, J. I. B. Wilson, A. J. Flewitt, W. I. Milne, and X. T. Zu, "Enhancement of microfluidic efficiency with nanocrystalline diamond interlayer in the ZnO-based surface acoustic wave device," Microfluid. Nanofluid. 15, 377 (2013).

${ }^{7}$ L. Schmid, D. A. Weitz, and T. Franke, "Sorting drops and cells with acoustics: Acoustic microfluidic fluorescence-activated cell sorter," Lab Chip 14, 3710 (2014)

${ }^{8}$ G. Destgeer, B. H. Ha, J. Park, J. H. Jung, A. Alazzam, and H. J. Sung, "Microchannel anechoic corner for size-selective separation and medium exchange via traveling surface acoustic waves," Anal. Chem. 87, 4627 (2015).

${ }^{9}$ R. J. Shilton, M. Travagliati, F. Beltram, and M. Cecchini, "Nanoliter-droplet acoustic streaming via ultra high frequency surface acoustic waves," Adv. Mater. 26, 4941 (2014).

${ }^{10}$ D. J. Collins, Z. Ma, and Y. Ai, "Highly localized acoustic streaming and size-selective submicrometer particle concentration using high frequency microscale focused acoustic fields," Anal. Chem. 88, 5513 (2016).

${ }^{11}$ K. D. Frampton, S. E. Martin, and K. Minor, "The scaling of acoustic streaming for application in micro-fluidic devices," Appl. Acoust. 64, 681 (2003).

${ }^{12}$ M. Alghane, Y. Q. Fu, B. X. Chen, Y. Li, M. P. Y. Desmulliez, and A. J. Walton, "Frequency effect on streaming phenomenon induced by Rayleigh surface acoustic wave in microdroplets," J. Appl. Phys. 112, 084902 (2012)

${ }^{13}$ M. Alghane, Y. Q. Fu, B. X. Chen, Y. Li, M. P. Y. Desmulliez, and A. J. Walton, "Scaling effects on flow hydrodynamics of confined microdroplets induced by Rayleigh surface acoustic wave," Microfluid. Nanofluid. 13, 919 (2012).

${ }^{14}$ T. Frommelt, D. Gogel, M. Kostur, P. Talkner, P. Hanggi, and A. Wixforth, "Flow patterns and transport in Rayleigh surface acoustic wave streaming: Combined finite element method and raytracing numerics versus experiments," IEEE Trans. Ultrason. Ferroelectrics Freq. Control 55, 2298 (2008).

${ }^{15}$ F. Kiebert, S. Wege, J. Massing, J. König, C. Cierpka, R. Weser, and H. Schmidt, "3D measurement and simulation of surface acoustic wave driven fluid motion: A comparison," Lab Chip 17, 2104 (2017).

${ }^{16}$ C. Devendran, T. Albrecht, J. Brenker, T. Alan, and A. Neild, "The importance of travelling wave components in standing surface acoustic wave (SSAW) systems," Lab Chip 16, 3756 (2016).

${ }^{17}$ M. B. Dentry, L. Y. Yeo, and J. R. Friend, "Frequency effects on the scale and behavior of acoustic streaming," Phys. Rev. E 89, 013203 (2014).

${ }^{18}$ J. Vanneste and O. Bühler, "Streaming by leaky surface acoustic waves," Proc. R. Soc. A 467, 1779 (2011).

${ }^{19}$ H. Antil, A. Gantner, R. H. W. Hoppe, D. Köster, K. Siebert, and A. Wixforth, Domain Decomposition Methods in Science and Engineering XVII (Springer, Berlin, Heidelberg, 2008), pp. 305-312.

${ }^{20}$ W. L. M. Nyborg, in Physical Acoustics, edited by W. P. Mason (Academic Press, 1965), pp. 265-331. 
${ }^{21}$ S. J. Lighthill, “Acoustic streaming," J. Sound Vib. 61, 391 (1978).

${ }^{22}$ J. Friend and L. Y. Yeo, "Microscale acoustofluidics: Microfluidics driven via acoustics and ultrasonics," Rev. Mod. Phys. 83, 647 (2011).

${ }^{23}$ Y. Q. Fu, J. K. Luo, X. Y. Du, A. J. Flewitt, Y. Li, G. H. Markx, A. J. Walton, and W. I. Milne, "Recent developments on $\mathrm{ZnO}$ films for acoustic wave based bio-sensing and microfluidic applications: A review," Sens. Actuators, B 143, 606 (2010).
${ }^{24}$ J. Reboud, Y. Bourquin, R. Wilson, G. S. Pall, M. Jiwaji, A. R. Pitt, A. Graham, A. P. Waters, and J. M. Cooper, "Shaping acoustic fields as a toolset for microfluidic manipulations in diagnostic technologies," Proc. Natl. Acad. Sci. U. S. A. 109, 15162 (2012).

${ }^{25}$ J. Eisener, A. Lippert, T. Nowak, C. Cairós, F. Reuter, and R. Mettin, "Characterization of acoustic streaming beyond $100 \mathrm{MHz}$," Phys. Procedia 70, 151 (2015). 